NOTE

\title{
Distinction between effective pattern-based and selection-based biodiversity surrogates is essential: caveats for managers
}

\author{
Serge Andréfouët ${ }^{1, *}$, Mélanie A. Hamel ${ }^{1,2}$, Mayeul Dalleau ${ }^{1,3}$ \\ ${ }^{1}$ Institut de Recherche pour le Développement, Centre de Nouméa, BP A5, 98848, Nouméa, New Caledonia \\ ${ }^{2}$ Present address: Australian Research Council Centre of Excellence for Coral Reef Studies, James Cook University, \\ Townsville, Queensland 4811, Australia \\ ${ }^{3}$ Present address: Kélonia, 46 rue du Général de Gaulle, 97436 Saint Leu, La Réunion — France
}

\begin{abstract}
Ecological concepts and data may justify the selection of biodiversity conservation plans among different options, but can also lead to poor conservation guidelines if not properly used. The use and meaning of the 'surrogate' concept in ecology and conservation planning contexts is a typical example. Surrogates are entities such as species, environmental variables or habitats, which are used to represent a target entity such as genes, species, ecosystems or related metrics. 'Pattern-based surrogacy' identifies effective surrogates using statistical congruence, while 'selection-based surrogacy' identifies effective surrogates through a notional conservation plan or prioritization analysis where sites are added to a set of protected areas, often using a complementarity criterion between sites. With this clear framework in mind, an investigation of the coral reef literature revealed that most published studies on surrogates of reef biodiversity refer in fact to pattern-based surrogates. Fundamentally, there is nothing wrong with both approaches. However, efficient pattern-based surrogates are often recommended for conservation planning, implying that they could be efficient selection-based surrogates. In fact, efficient pattern-based surrogates are not necessarily efficient selection-based surrogates and vice versa. The reason is the complementarity rules used by selection algorithms. We call for more clarity from authors on the context in which effective surrogates have to be used, and more caution from managers when surrogates are to be used in conservation plans.
\end{abstract}

KEY WORDS: Surrogacy · Surrogate effectiveness - Remote sensing · Conservation planning · Habitat map · Coral reefs

Resale or republication not permitted without written consent of the publisher

\section{INTRODUCTION}

Ecology aims to describe and study distribution and patterns of biodiversity to empirically infer the complex bio-physico-chemical processes that have created this biodiversity through time and space. Conservation science and management then use ecological descriptions and patterns to elaborate biodiversity conservation plans. Ecologists increasingly turn to applied conservation science given the increasing societal (and funding) requirements for 'use- ful' research to achieve population well being and resource sustainability. In fact, conservation is rewarding for ecologists as citizens worried about how the natural world is degrading. But also intellectually speaking since conservation brings new challenges and fresh paradigms when looking at classic ecological problems and data sets. However, in some instances, conservation has brought specific angles to ecological problems to the point that it may be necessary to clarify recent concepts (Caro 2010). Confusing recommendations may have negative and costly con- 
sequences on the decision-making process because managers could channel substantial efforts and funds in the wrong directions (Carwardine et al. 2008).

The goal of this Note is to (re-)emphasize the specific use and meaning of the words 'surrogacy' and 'surrogates' according to their common uses in ecology and conservation planning. In its broader ecological meaning, surrogates can be defined as entities (species, environment data, habitats) which are used to represent other biodiversity entities (genes, species, ecosystems or biodiversity metrics). The practical justification of using surrogates generally comes from the substantial funds, expertise and time needed to acquire new data (Grantham et al. 2008, 2009). Instead, existent data or data that are cheaper or easier to acquire (i.e. surrogates) are used. In the conservation planning literature, reference to a conservation plan is implicit. For Caro (2010) for instance, surrogates are entities which are used to represent other species or aspects of the environment to attain a conservation objective. The use of surrogate data sets instead of more precise ideal biological data sets is increasing (e.g. Mellin et al. 2011). However, under the general aforementioned 'surrogate'/ 'surrogacy' vocabulary, there are several distinct notions. Since they may lead to paradoxical and apparently contradictory guidelines in terms of data acquisition and conservation strategies, managers need to be aware of the differences.

\section{PATTERN-BASED AND SELECTION-BASED SURROGATES IN ECOLOGY AND CONSERVATION}

In all terrestrial, marine and freshwater realms, many ecological studies have investigated the links between biodiversity patterns (e.g. exhaustive species diversity) and ancillary variables (typically environmental and habitat variables, but also other accessible species and higher-order taxa). The goal is to infer the processes explaining the observed biodiversity, but also to find good direct predictors of biodiversity patterns (Gaston 2000, Ricklefs 2004, Tittensor et al. 2010). In ecology, if that link is statistically strong, the ancillary variable can be used as a proxy of the studied biological system, especially if this proxy is easier, faster or cheaper to study than the initial target. In this case, simple congruence measurements between the 2 variables (such as Spearman and Kendall correlation coefficients or Mantel tests) are sufficient to estimate the effectiveness of the proxy. Knowledge of biodiversity from genes to ecosystems is far from perfect in most locations. Collecting new adequate comprehensive gapfilling data is considerably demanding in terms of resources and expertise (Balmford \& Gaston 1999). Therefore, predictive ecological models that integrate efficient proxies are useful cost-effective tools to describe biological patterns (Mellin et al. 2011). In ecology, these proxies are frequently called 'surrogates'. In these types of applications, these surrogates are used as statistical 'predictors' or 'indicators' of other biodiversity features (for which data are limited). Such surrogates have recently been described as 'pattern-based surrogates' (Grantham et al. 2010).

In systematic conservation planning (sensu Margules \& Pressey 2000), the goal is often to produce an efficient strategy of prioritization, from a selection of different scenario outputs. Scenarios aim to reach a specific conservation target, such as protecting a maximum of all biological features over a certain area. Often, adequate knowledge is limited (Rodrigues \& Brooks 2007), and conservation planners must use proxies instead of the ideal data sets to run their scenario (Caro 2010). For instance, the aim of managers can be to include the greatest number of species encountered in one area in a reserve system covering a portion of this area. If, for instance, fish biodiversity is deemed suitable to represent the total biodiversity in this area, conservation planners may be tempted to build their conservation strategy on fish biodiversity distribution. In this case, fish distribution would be a 'surrogate' of the (unknown) total biodiversity in the conservation design. Another example could be to use habitat maps as surrogates of species (Dalleau et al. 2010). In that case, the surrogacy scenario would include a minimal proportion (area) of all habitats in a reserve system. It would then be compared to a reference scenario targeting the inclusion of all recorded species into the system. There are different methods to assess the effectiveness of selection-based surrogates, all based on a comparison between the ability of the design method to include the feature(s) of interest in the system when using the surrogate and when using the specified reference (see Grantham et al. 2010 for a description of the main methods). Typically, if the habitat-based reserve system includes a similar number of species within a similar number of protected units in the set of reserves, compared to the speciesbased reserve system, the habitat can be considered an efficient surrogate of total biodiversity from a conservation planning standpoint. The effective accumulation of the targeted species in the set of reserves can be measured with accumulation curves (Sarkar 
et al. 2005), and related metrics (such as the species accumulation index, SAI; Rodrigues \& Brooks 2007). The SAI is based on the differences of the areas under the accumulation curves. It quantifies the effectiveness of the scenario, and thus the effectiveness of the used surrogate. In general, a surrogate will be more efficient than another if conservation scenario outputs show that a conservation target is reached at a lower cost than with other datasets. Therefore, the measure of surrogacy potential in a conservation context is not based solely on a statistical measure of good fit, but it depends on the costeffectiveness of a scenario, which is itself highly dependent on prioritization methods. In most cases, these prioritization methods are selection or optimization algorithms (Moilanen et al. 2009). Such surrogates are known as 'selection-based surrogates' (Grantham et al. 2010).

It is also important to add that, often, 'patternbased' and 'selection-based' surrogates are the same entities (for instance a habitat descriptor). It is the evidence of effectiveness, and how it is measured, that makes them pattern-based or selection-based, and not the nature of the entity.

\section{CONTRASTED EFFECTIVENESS OF PATTERN-BASED AND SELECTION-BASED SURROGATE LEADS TO APPARENT PARADOX}

What is often seen as a paradox is that an efficient pattern-based surrogate in ecology is not necessarily an efficient selection-based surrogate in conserva- tion. The opposite is also verified. For instance, if a surrogate has a strong statistical spatial link with the distribution of a suite of endangered rare species, depending on the scenario used to save these species, the use of the surrogate instead of the actual list of species can be either efficient or misleading. Examples are found in the literature. For coral reefs, Beger et al. (2007), who investigated cross-taxa surrogacy, gave a good example that contradicts the idea that a good pattern-based surrogate is a good selection-based surrogate. They showed that even though there is a spatial correlation between a variable $X$ (fish, corals or molluscs) diversity and a variable $Y$ (fish, corals, molluscs) diversity, the design of a marine protected area based on $X$ would lead to a poor representation of $Y$ diversity. On the other hand, Dalleau et al. (2010) showed that using marine benthic habitats to design a marine protected area would likely benefit species diversity of various taxa despite no apparent spatial correlation. This demonstrates the point that efficient selection-based surrogates are compatible with low spatial congruence and weak efficiency as pattern-based surrogates. This paradox is of course only apparent.

Of this pseudo-paradox, 2 illustrations are given here using a simple simulation and a case study. (1) For 2 simulated data sets (Fig. 1), we show the possible occurrence of strong and weak patternbased effectiveness, associated with respectively weak and strong selection-based effectiveness (Fig. 2 and Table 1). (2) We extracted a subset of the data set presented by Dalleau et al. (2010), who tested coral reef habitat maps as surrogates of spe- a) Fictive dataset $1 \quad \square$ Presence $\square$ Absence

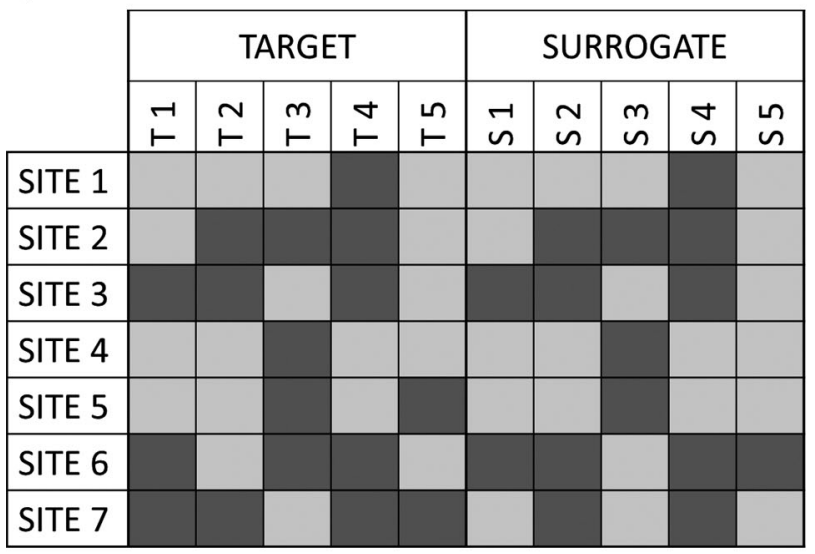

b) Fictive dataset 2

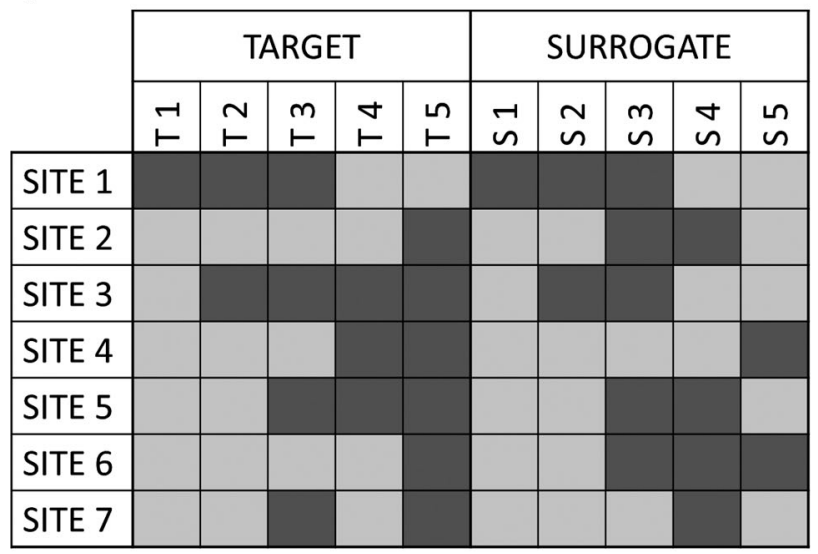

Fig. 1. Fictive datasets used to illustrate effective (a) pattern-based surrogates which are not effective selection-based surrogates and (b) selection-based surrogates which are not effective pattern-based surrogates (cf. Fig. 2 and Table 1). The matrix indicates the distribution (presence/absence) of 5 surrogate features (S1, S2, S3, S4, S5) and 5 target features (T1, T2, T3, T4, T5) among 7 sites. Perfect spatial congruence would be evidenced as a perfect match between locations 


\section{a) Fictive dataset 1}

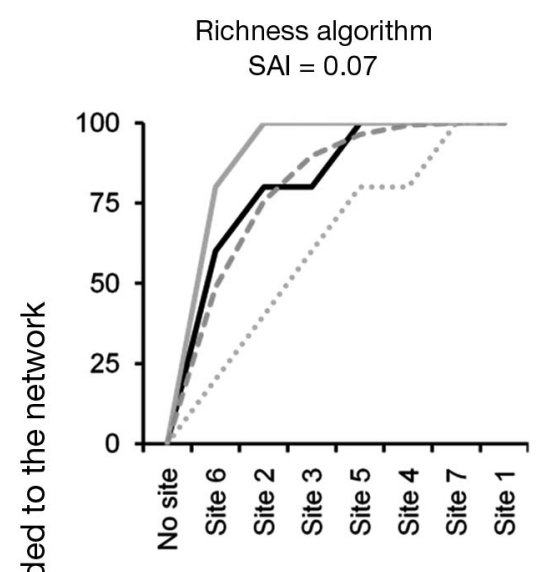

b) Fictive dataset 2

Richness algorithm $\mathrm{SAl}=0.45$

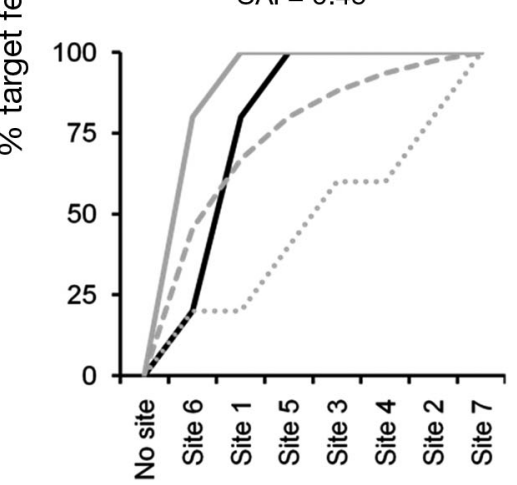

Rarity algorithm $\mathrm{SAl}=-0.30$

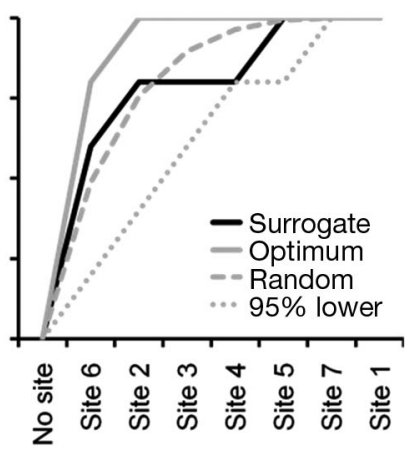

Sites added to the network

Fig. 2. Selection-based surrogacy effectiveness measured for fictive datasets 1 and 2 . Two iterative reserve design algorithms were tested, maximizing either richness and complementarity or rarity and complementarity of surrogates within the selected set of reserves. This means that sites that maximized the surrogate richness (respectively rarity), were added one by one to the set of reserves. To satisfy the complementarity criterion, sites containing surrogates not yet included in the set of reserves were prioritized. The first site included in the set of reserves was the site with the richest (respectively rarest) set of surrogates. During the iterative process, the representation of the target within the selected set of reserves was monitored. The rate of target inclusion was subsequently compared with the rate of target inclusion obtained when selecting sites randomly: 1000 random simulations yielded a mean and confidence interval (mean + $1.96 \mathrm{SE}$ ). A reference optimal scenario was also built using the same algorithms, selecting sites directly regarding the target data. Plots of target representation against the number of sites added are called accumulation curves. Species accumulation indices (SAI, Rodrigues \& Brooks 2007) were calculated to assess more accurately the position of the 'surrogate' curve regarding the 'optimum' curve and the 'random' curve. (a) Evidence that a strong pattern-based surrogate (cf. Fig. 1a and Table 1) can be a weak selection-based surrogate. (b) Evidence that a strong selection-based (cf. Fig. 1b and Table 1) surrogate can be a weak pattern-based surrogate

cies inventories in Wallis Island (South Pacific). Dalleau et al. (2010) reported a poor correlation (non-significant) between macroalgal diversity and habitat diversity at all spatial scales and for all levels of habitat characterization (Fig. 3). Yet, habitats were always effective surrogates in representing the same algal diversity in a conservation plan (Fig. 4). These 2 examples highlight the common occurrence of the paradox.

In the recent coral reef literature (reviewed below) and elsewhere, the word 'surrogate' is often used with a 'patternbased' meaning. There are many claims that good surrogates (sensu pattern-based) are identified, but conclusions on effectiveness of pattern-based surrogacy cannot be systematically generalized to selectionbased surrogacy. This caveat is important for managers and funding agencies. If they aim for a conservation objective using decision support methods such as systematic conservation planning, they should fund studies that are articulated around a conservation plan, and not ecological studies that statistically quantify links between variables. This plan can be quite specific, as the local conservation objectives can also be quite specific as well. Therefore, using the published pattern-based conclusions from one site (or region), to identify a conservation area network for another site (or region), is not a recommended practice.

As mentioned by Grantham et al. (2010, p. 8), 'insights into surrogates could be gained from reviewing aspects of the ecology and biogeography of species that both support the use of surrogates', or in other words, it is useful to identify beforehand the specific aspects of species (e.g. their rarity) that make surrogates effective or ineffective. This also suggests that demonstrated efficient pattern-based surrogates could be used to develop selection-based surrogacy strategies. But, as highlighted here, effectiveness of both cannot be guaranteed. Methods for assessing the effectiveness of pattern-based and selectionbased surrogates are different, which makes both types of surrogates not necessarily transposable. According to Williams et al. (2006), who discussed this in depth, 
Table 1. Pattern-based surrogacy effectiveness measured for fictive datasets 1 and 2 (Fig. 1). Pattern-based surrogate effectiveness is measured with non-parametric tests based on rank correlation: Mantel test (correlation between target and surrogate composition), Spearman test (relationship between target and surrogate richness) and Pearson test (linear relationship between target and surrogate richness). The different metrics show that the surrogates in datasets 1 and 2 are, respectively, good and poor pattern-based surrogates

\begin{tabular}{|lcc|}
\hline & Dataset 1 & Dataset 2 \\
\hline $\begin{array}{l}\text { Mantel test } \\
\text { Test statistic } \\
\text { Spearman test } \\
\text { Rho }\end{array}$ & 0.82 & 0.20 \\
$\begin{array}{l}\text { Pearson test } \\
\text { Correlation coefficient }\end{array}$ & 0.71 & 0 \\
\hline
\end{tabular}

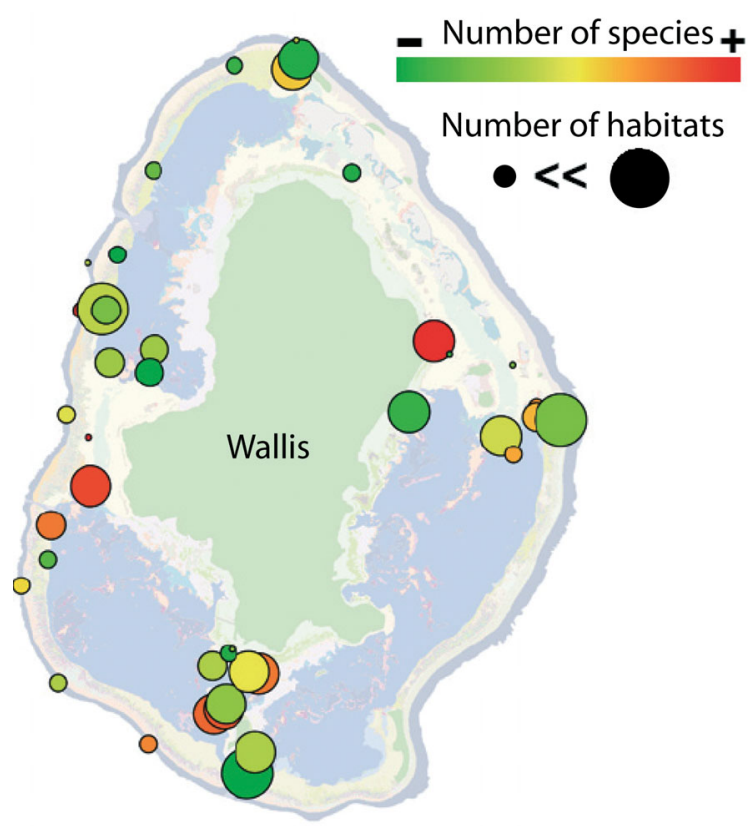

Kendall correlation $=0.11$

Fig. 3. From Dalleau et al. (2010), a real-case study in Wallis Island (South Pacific) demonstrating that good selectionbased surrogates can be poor pattern-based surrogates. Targets are macroalgae species, and surrogates are habitats. In this example, we used a habitat geomorphological map (map C in Dalleau et al. 2010) and a scale of analysis of $250 \mathrm{~m}$ to compute habitat richness around the stations. Circle size indicates species richness at each census station, while colour indicates habitat richness in the considered neighbourhood. Good congruence would result in small green circles, medium-sized congruence in yellow and orange, and poor congruence in large red circles. A poor correlation was measured between target and surrogate richness, with low Kendall correlation $\tau=0.11(\mathrm{p}=0.19)$. This poor correlation was the best achieved for the macroalgae taxa, for all types of habitat maps and scales (Dalleau et al. 2010)
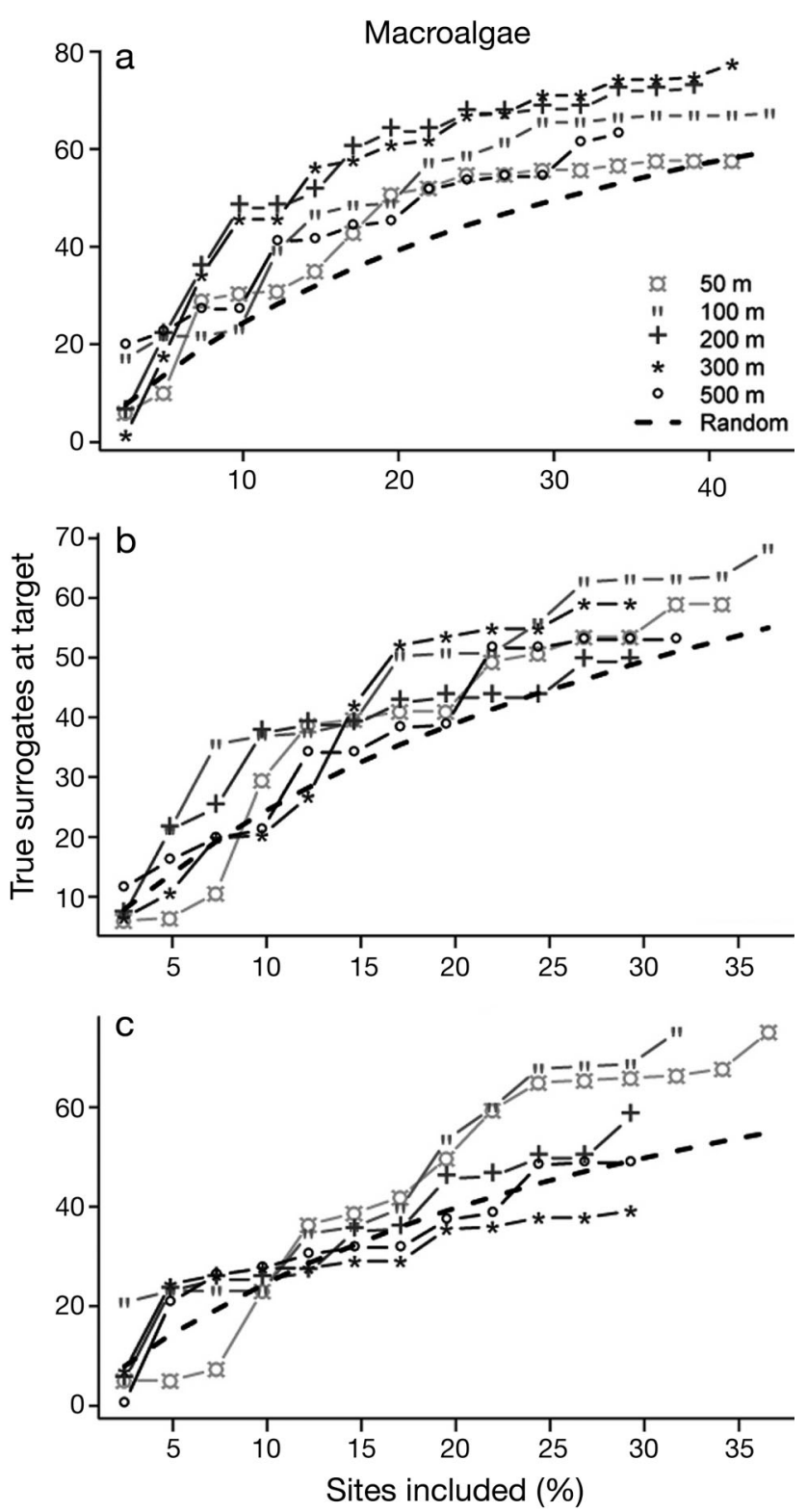

Fig. 4. Using a rarity-complementarity algorithm, and various types of habitat maps and scales, almost all accumulation curves (as explained in Fig. 2) are above the reference random curve (which here is the upper level of the $95 \%$ confidence interval achieved after 1000 random scenarios). This indicates good effectiveness of habitats as surrogates of macroalgae with this prioritization design. Results for the various scales are shown for (a) a fine benthic habitat map, (b) a fine geomorphological map, and (c) a coarse geomorphological map. See Dalleau et al. (2010) for details

the important criteria to achieve effectiveness of a set of reserves when using surrogates is not the strength of the relationship between biodiversity patterns of the target and its surrogate (pattern-based surrogate) but biotic complementarity among areas. Comple- 
mentarity was redefined as 'a measure of the extent to which an area or set of areas, contributes unrepresented features to an existing area or set of areas' (Margules \& Pressey 2000, p. 249). Complementarity analysis is thus a function of an iterative process that selects management units in a stepwise fashion such that each new unit contributes best to the overall criteria (e.g. maximum representation of species; Caro 2010). Fig. 1a clearly suggests that patterns of complementarity need to be considered before using pattern-based surrogates for selection-based surrogates.

\section{THEMATIC EXAMPLES OF PATTERN-BASED AND SELECTION-BASED SURROGATES FROM THE CORAL REEF LITERATURE}

Coral reefs are globally at risk, threatened by climate change and anthropogenic disturbances, and conservation actions are exponentially increasing. This is paralleled by a similar increase in coral reef conservation literature, including a growing use of the concept of surrogacy applied to coral reefs. As such it is a good ecosystem case study to illustrate some misuses of the concepts and definitions presented above. Thus, we did not conduct a comprehensive review of the use of biodiversity surrogates (see instead Ferrier 2002, Favreau et al. 2006, Rodrigues \& Brooks 2007) but highlighted representative current references to the surrogacy concept.

In early 2011, we performed a search on surrogacy papers from the ISI Web of Science literature database using a combination of the following key words: coral, reef, surrogate, surrogacy. For each study, we analysed the conservation target, the potential surrogate feature, the vocabulary used when referring to the surrogate and the aim or context of the study. Here, the terms 'conservation target' and 'target feature' refer to the attribute of biodiversity on which conservation actions or protection measures focus.

We recorded 6 papers using the generic term 'surrogate' that actually referred to pattern-based surrogates (Table 2). These papers represented studies that used surrogates to (1) describe biodiversity patterns; (2) estimate biodiversity metrics (e.g. species or habitat richness, diversity); (3) develop models to predict the spatial distribution of conservation targets (Moilanen et al. 2009, McArthur et al. 2010). These studies suggested, as perspectives, that the resulting data and maps could be used for rapid biodiversity assessments and for integration in conservation planning schemes. Most of the studies assessed the potential of habitats and environmental variables to predict reef fish diversity, except De Troch et al. (2008), who demonstrated that higher taxa in copepods and nematodes can be used as pattern-based surrogates for species-level identifications or fast assessments. One interesting example is from Dunn \& Halpin (2009). They suggested using 'rugosity' as a pattern-based surrogate of hard-bottom habitats, which are known to support high levels of biodiversity. They then proposed to use hardbottom habitat predictions from the rugosity as input of siting algorithms to protect fish richness, and more generally marine biodiversity. Lindsay et al. (2008) investigated the potential of habitats as patternbased surrogates to predict fish assemblages in a marine protected area. Harborne et al. (2008) assessed the pattern-based surrogacy potential of habitat heterogeneity as a surrogate of biodiversity to support its use as a selection-based surrogate. Anderson \& Yoklavich (2007) and Anderson et al. (2009) proposed to use their efficient pattern-based surrogates (seabed habitats) for management purposes. Wedding et al. (2008) stated that since there was a strong link between the pattern-based surrogate (substrate rugosity) and the considered ultimate conservation target (reef fish assemblages), the surrogate could be used to assist in prioritizing areas for conservation and management but gave no further details on how this could be applied. It is obvious in the coral reef literature that there is a propensity to consider pattern-based surrogates for further conservation planning. However, the message remains unclear concerning the exact methods to apply.

Few selection-based surrogacy studies (Table 1) appear in the coral reef literature. They focused on (1) testing selected taxa as surrogates of total reef biodiversity (Beger et al. 2007), (2) testing of selected taxa as surrogates of other selected taxa (Beger et al. 2003, 2007), and (3) testing coral reef habitats as surrogates of taxa, ecological functions and ecosystem services (Mumby et al. 2008, Dalleau et al. 2010). Generally, these studies have avoided mixing the selection-based and pattern-based concepts, and even clearly pointed out the paradox (Beger et al. 2007, Dalleau et al. 2010).

In the recent coral reef literature, the word 'surrogate' is thus often used with a 'pattern-based' meaning (Table 2). Many claims that good surrogates (sensu pattern-based) were identified, but conclusions on efficiency of pattern-based surrogacy cannot be simply transferred to selection-based surrogacy. Thus, managers should be careful if they aim to identify a set of reserves using a previously identified pattern-based surrogate. 


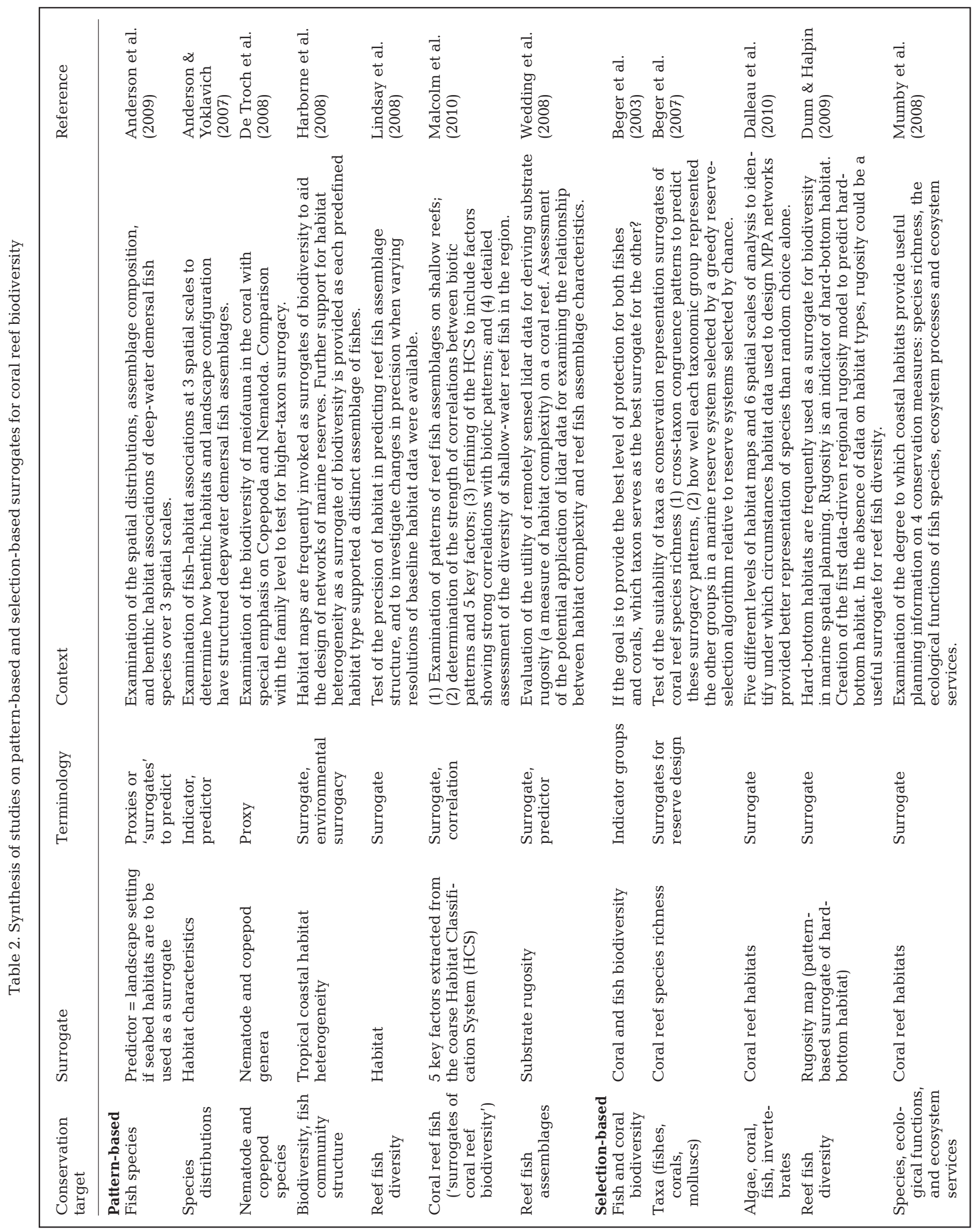




\section{CONCLUSION}

In their review on the effectiveness of surrogates in conservation planning, Rodrigues \& Brooks (2007) clearly made the distinction between the different types of surrogacy approaches by examining studies using reserve selection, and excluding those testing correlations between surrogates and target features. The 2 different ways of using the terms 'surrogate' or 'surrogacy' pointed out by Rodrigues \& Brooks (2007) are recurrent as shown by our coral reef review, and this has led to confusion, contrasting and apparently opposite results, and possible misunderstandings of the concepts and methods applied.

Pattern-based surrogates are mostly descriptive tools, whereas selection-based surrogates are strictly conservation tools. Managers have to keep in mind that a good pattern-based surrogate is not necessarily a good selection-based surrogate and vice versa.

Conceptually, there is inherently nothing wrong in using one approach or the other for a given problem. However, we recommend that authors clarify upfront which approach they use and provide a clear framework to avoid misleading conservation advice. We also believe that systematic presentation would facilitate future inter-study comparisons, meta-analysis and the establishment of clear guidelines.

Acknowledgements. This paper benefited from the comments made by 3 anonymous reviewers. C. Payri provided the macroalgae data used in Figs. $3 \& 4$.

\section{LITERATURE CITED}

Anderson TJ, Yoklavich MM (2007) Multiscale habitat associations of deepwater demersal fishes off central California. Fish Bull 105:168-179

Anderson TJ, Syms C, Roberts DA, Howard DF (2009) Multiscale fish-habitat associations and the use of habitat surrogates to predict the organisation and abundance of deep-water fish assemblages. J Exp Mar Biol Ecol 379: 34-42

Balmford A, Gaston KJ (1999) Why biodiversity surveys are good value. Nature 398:204-205

> Beger M, Jones GP, Munday PL (2003) Conservation of coral reef biodiversity: a comparison of reserve selection procedures for corals and fishes. Biol Conserv 111:53-62

- Beger M, McKenna SA, Possingham HP (2007) Effectiveness of surrogate taxa in the design of coral reef reserve systems in the Indo-Pacific. Conserv Biol 21:1584-1593

Caro TM (2010) Conservation by proxies: indicator, umbrella, keystone, flagship, and other surrogate species. Island Press, Washington, DC, USA

Carwardine J, Wilson KA, Watts M, Etter A, Klein CJ, Possingham HP (2008) Avoiding costly conservation mistakes: the importance of defining actions and costs in spatial priority setting. PLoS ONE 3:e2586
Dalleau M, Andréfouët S, Wabnitz CCC, Payri C and others (2010) Use of habitats as surrogates of biodiversity for efficient coral reef conservation planning in Pacific Ocean Islands. Conserv Biol 24:541-552

> De Troch M, Raes M, Muthumbi A, Gheerardyn H, Vanreusel A (2008) Spatial diversity of nematode and copepod genera of the coral degradation zone along the Kenyan coast, including a test for the use of higher-taxon surrogacy. Afr J Mar Sci 30:25-33

$>$ Dunn DC, Halpin PN (2009) Rugosity-based regional modeling of hard-bottom habitat. Mar Ecol Prog Ser 377:1-11

> Favreau JM, Drew CA, Hess GR, Rubino MJ, Koch FH, Eschelbach KA (2006) Recommendations for assessing the effectiveness of surrogate species approaches. Biodivers Conserv 15:3949-3969

Ferrier S (2002) Mapping spatial pattern in biodiversity for regional conservation planning: Where to from here? Syst Biol 51:331-363

Gaston KJ (2000) Global patterns in biodiversity. Nature 405:220-227

Grantham HS, Moilanen A, Wilson KA, Pressey RL, Rebelo TG, Possingham HP (2008) Diminishing return on investment for biodiversity data in conservation planning. Conserv Lett 1:190-198

Grantham HS, Wilson KA, Moilanen A, Rebelo T, Possingham HP (2009) Delaying conservation actions for improved knowledge: How long should we wait? Ecol Lett 12:293-301

Grantham HS, Pressey RL, Wells JA, Beattie AJ (2010) Effectiveness of biodiversity surrogates for conservation planning: Different measures of effectiveness generate a kaleidoscope of variation. PLoS ONE 5:e11430

> Harborne AR, Mumby PJ, Kappel CV, Dahlgren CP, Micheli F, Holmes KE, Brumbaugh DR (2008) Tropical coastal habitats as surrogates of fish community structure, grazing, and fisheries value. Ecol Appl 18:1689-1701

Lindsay MJ, Patterson HM, Swearer SE (2008) Habitat as a surrogate measure of reef fish diversity in the zoning of the Lord Howe Island Marine Park, Australia. Mar Ecol Prog Ser 353:265-273

Malcolm HA, Smith SDA (2010) Objective selection of surrogate families to describe reef fish assemblages in a subtropical marine park. Biodivers Conserv 19:3611-3618

Margules CR, Pressey RL (2000) Systematic conservation planning. Nature 405:243-253

McArthur MA, Brooke BP, Przeslawski R, Ryan DA and others (2010) On the use of abiotic surrogates to describe marine benthic biodiversity. Estuar Coast Shelf Sci 88: 21-32

> Mellin C, Delean S, Caley MJ, Edgar GJ and others (2011) Effectiveness of biological surrogates for predicting patterns of marine biodiversity: a global meta-analysis. PLoS ONE 6:e20141

Moilanen A, Wilson KA, Possingham H (2009) Spatial conservation prioritization. Quantitative methods and computational tools. Oxford University Press, Oxford

Mumby PJ, Broad K, Brumbaugh DR, Dahlgren CP and others (2008) Coral reef habitats as surrogates of species, ecological functions, and ecosystem services. Conserv Biol 22:941-951

> Ricklefs RE (2004) A comprehensive framework for global patterns in biodiversity. Ecol Lett 7:1-15

> Rodrigues ASL, Brooks TM (2007) Shortcuts for biodiversity conservation planning: the effectiveness of surrogates. Annu Rev Ecol Evol Syst 38:713-737 
Sarkar S, Justus J, Fuller T, Kelley C, Garson J, Mayfield M (2005) Effectiveness of environmental surrogates for the selection of conservation area networks. Conserv Biol 19: 815-825

Tittensor DP, Mora C, Jetz W, Lotze HK, Ricard D, Vanden Berghe E, Worm B (2010) Global patterns and predictors of marine biodiversity across taxa. Nature 466: 1098-1101

Editorial responsibility: Hans Heinrich Janssen, Oldendorf/Luhe, Germany
Wedding LM, Friedlander AM, McGranaghan M, Yost RS, Monaco ME (2008) Using bathymetric lidar to define nearshore benthic habitat complexity: implications for management of reef fish assemblages in Hawaii. Remote Sens Environ 112:4159-4165

Williams P, Faith D, Manne L, Sechrest W, Preston C (2006) Complementarity analysis: mapping the performance of surrogates for biodiversity. Biol Conserv 128:253-264

Submitted: July 25, 2011; Accepted: February 8, 2012 Proofs received from author(s): April 18, 2012 